\title{
Small volume mechanical response insights: revealed contributions in advancing high mechanical performance
}

\author{
Y. Katz \\ Negba, Beer-Sheva 84230, Israel
}

\begin{abstract}
Three factors have emerged in order to upgrade the understanding of ultra small volume mechanical behavior in terms of multi-scale analysis and models. Firstly, the global/local approach based on physical models formulation, secondly, the continuous effort vis-à-vis microelectronic requirements for effective material performance and thirdly, the remarkable progress in experimental capacity that requires ultra-fine features visualization and measurements beside computational abilities. The current study is centered on iron based silicon polycrystalline alloy and the mechanical characterization of $20-50 \mathrm{~nm}$ silicon particles by contact methodology at ambient temperatures. In both, either monotonic or cyclic, mechanical properties were size dependent. Regarding the iron-silicon single crystals, mini compact tension specimens enabled the tracking of crack-tip dislocation emissions and their interactions in order to deepen the shielding potential exploration. Deformation/fracture processes and ductile-brittle fracture models could be elaborated. The aforementioned examples in elastic-plastic crystals were still intended to emphasize conceptual insights. Higher mechanical performance is examined from emerging localized findings and nanomechanical response analysis.
\end{abstract}

Keywords: silicon, iron-based silicon, single crystals, contact tests, fracture mechanics, nano mechanical response.

\section{Introduction}

The practical sense of high performance materials deserves further elaboration. Still on a practical level, material is evaluated by optimizing the exact functional properties for certain applications. However, structural integrity or lifetime 
evaluations are prerequisite requirements even in situations in which electrical, optical, magnetic and other properties become a major concern. Clearly other intrinsic/extrinsic variables are included in the material assessment as related to specific service conditions. Applications in marine, space, nuclear and off shore imply typical restriction as well as thermal, state of stress and rate variables that imposed additional windows. Thus, structural materials are screened in a comprehensive methodology beside economical impact. The relations between mechanical properties and microstructure have already established a sophisticated discipline. By definition, the scope is broadening to imperfections of structure and substructure affecting fracture resistance, fatigue, creep and interactive processes in service. It is the author's opinion that the most effective avenue to advance material performance remains in activities that can deepen the basic understanding in material response. Accordingly, the present investigation has been constructed by following novel techniques in single crystals. Engagement in small volume segments raises the scale effect in material performance. For solely high values of tensile strength, strong materials characterization has been addressed earlier $[1,2]$ in dedicated studies. However, even in the past, other engineering criteria aspects have not been excluded. The aforementioned contributions provided a good background in either materials such as whiskers, composites or hardening mechanisms, thermo-mechanical processing or line defect dynamics beside intensive investments of the crack behavior.

\section{Experimental procedures}

\subsection{The silicon particles case}

Identified nano spheres of silicon particles in the range of $20-50 \mathrm{~nm}$ in diameter have been selected. These particles were synthesized by injecting the vapor phase of silicon tetrachloride into argon-hydrogen thermal plasma that was expanded. The low-pressure stage through a nozzle drives the silicon nano particles. These hypersonic plasma particles were deposited on sapphire substrate in a line fashion. Isolated particles enabled the conduction of controlled tests in monotonic and cyclic imposed mechanical loads. The study was assisted by utilizing micro-probe microscope-based nano indentation. A nano indenter of $1 \mu \mathrm{m}$ tip radius was used for tests at an ambient temperature. The current combined program of imposed contact loads and visualization provided ultra fine features quantitative information. Due to the ultra fine particle size the indenter provided a nano compression condition similar to squeezing the particles in between two rigid platens. More about the experimental procedures was addressed by Rao et al [3], Gerberich et al [4] and elsewhere.

\subsection{The Fe-3\%wtSi single crystals cases}

Following the fracture mechanics methodology mini-disc pre-cracked compact tension specimens of Fe-3\%wtSi single crystals were tested (Fig. 1). The discs 


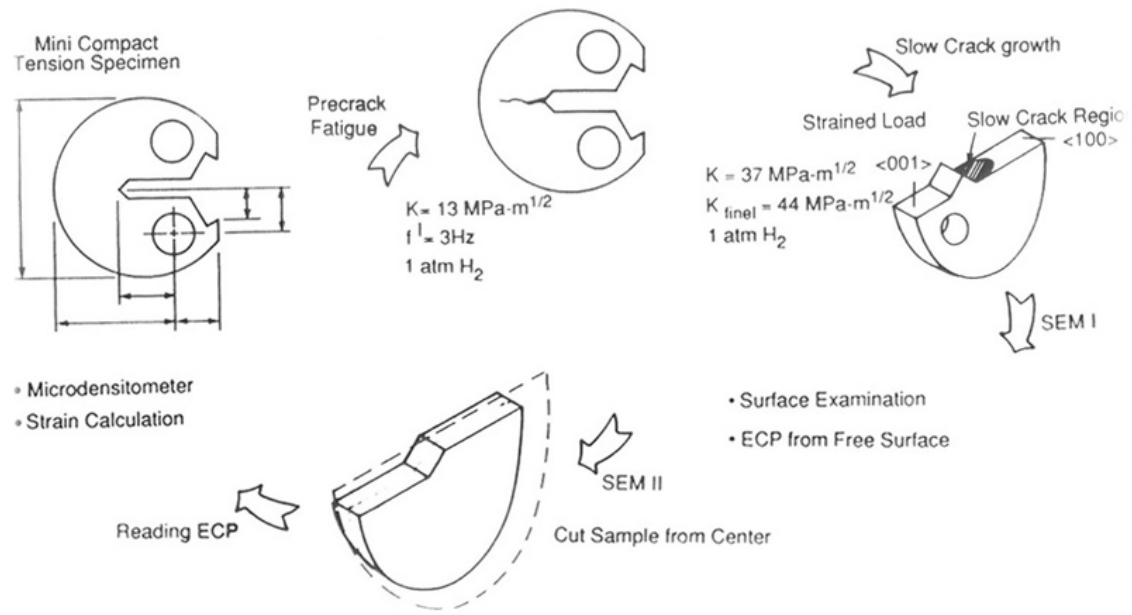

Figure 1: Specimens' geometry.

are about $22 \mathrm{~mm}$ in diameter and were calibrated by the compliance technique actually determined on iron specimens.

Pre-cracking was performed in the selected quasi-brittle material by compression-compression fatigue at temperatures below the ductile-brittle transition. By adopting this procedure, sharp crack-tips with a minimal either damaged zone or residual strain at the crack-tip become possible. The fracture mechanics tests used different crack orientations with typical crosshead rates of $15-10^{-6} \mathrm{~m} / \mathrm{s}$. Besides cyclic load tests, the specimens were also loaded to specific stress intensity factors and after unloading slip traces were examined as revealed on the external surfaces. Surface slip traces were tracked by using a Normaski contrasting light microscope as a first step in order to establish crystal plasticity habits. The mechanical response was supplemented by fractography using Scanning Electron Microscope (SEM) images. In addition, interferometry assisted by Zygo laser interferometer was utilized. In order to determine plastic deformation, particularly in the brittle regime, the Selected Area Electron Channeling technique (SAEC) was also added. Finally, dislocation structures were observed by a Transmission Electron Microscope (TEM) using thin foils from extracted small cylinders. From the specimens, material was cut out at specific locations by the electronic discharge machining technique. Here, locations approached the crack-tip that allowed critical information to be gathered. Surface slip traces were analyzed regarding the specimen crystallographic orientation and the crack system. Thus, observations could be compared to theoretical predictions. An important experimental conclusion emerged that in the investigated material, the very sharp crack-tip was achieved by compression-compression fatigue pre-cracking at $143 \mathrm{~K}$ and a load ratio of about 10 . More has been addressed previously [5-8]. 


\section{Experimental results}

\subsection{The silicon particles cases}

Under the present experimental conditions, small volume silicon particles as depicted in fig. 2, the bright field TEM diffraction is a selected ADP [112] zone axis.

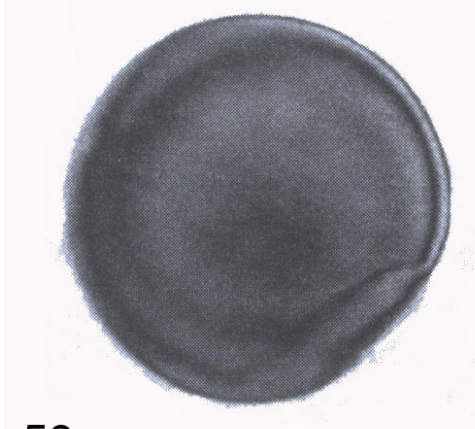

$50 \mathrm{~nm}$

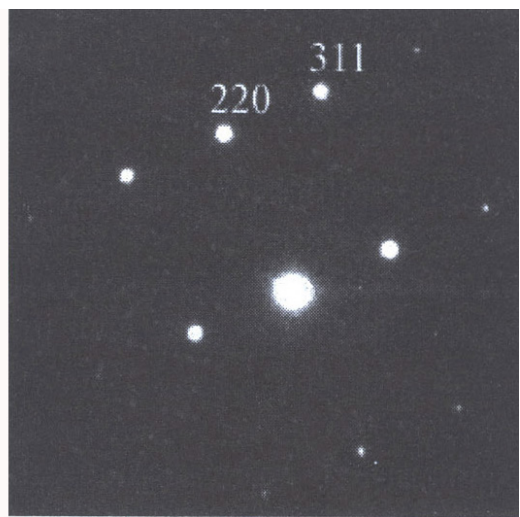

Figure 2: (Left) typical particle (right) bright field TEM.

Mechanically the particle resulted in significant higher hardness values as compared to bulk values. For example, for a $20 \mathrm{~nm}$ particle the typical hardness value exceeded $50 \mathrm{GPa}$ while a reduced value of about $10 \mathrm{GPa}$ was obtained for silicon wafer. If $R$ is the particle radius, the hardness was proportional to $1 / R^{2}$. The notion that silicon is considered to be brittle at temperatures below $773 \mathrm{~K}$ behaved in ambient temperatures in a typical elastic-plastic response with striking TEM results. The additional finding was important beyond the significant plasticity regime characterization. In particular, for repeated contact load static and dynamic work hardening alluded to the dislocation dynamic model in creating super hard material. In the cyclic response it became apparent that the partition between reversible and irreversible plasticity regime can be revealed quantitatively, with consistent behavior that remained size dependent (see fig. 3).

\subsection{The Fe-3\%wtSi single crystal cases}

Regarding the specimen with a $\{001\}<110>$ orientation that was tested in a monotonic rising load, the following was observed. The crack-tip opening continued to increase after yielding actually reaching an angle larger than $30^{\circ}$. Since severe plastic deformation occurred, the crack-tip region necked down dramatically with lateral contraction of about $85 \%$. However, marginal variations of the loading rate or overload fracture, caused transition to the brittle mode by cleavage. This is not surprising but another confirmation of a semi-brittle 


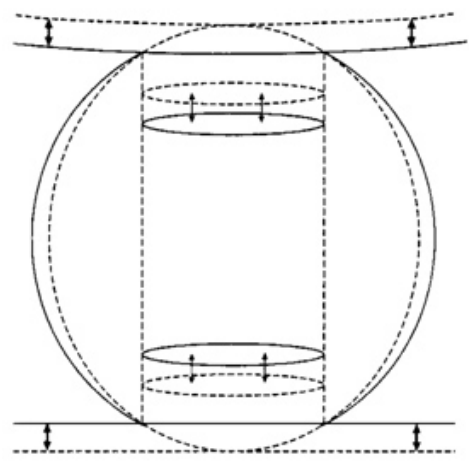

(a)

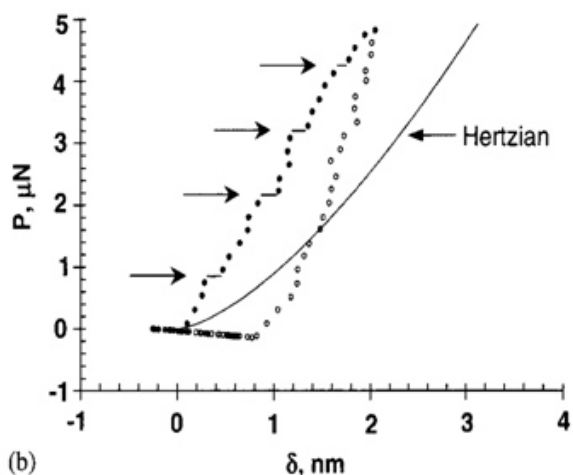

(b)

$\delta, \mathrm{nm}$

Figure 3: (a) Schematic-deformation by contact (b) Load displacement curve of a $38.6 \mathrm{~nm}$ particle.

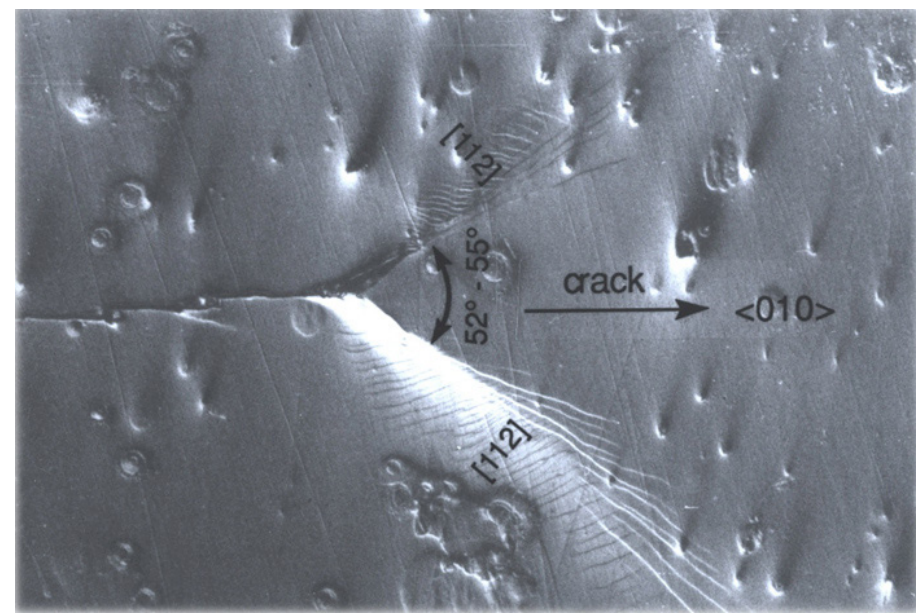

Figure 4: $\quad$ Slip traces on the side surface $(001)<010>$ crack system.

material behavior; namely, ductile/brittle transition is strain rate dependent. Thus dislocation nucleation or movements have a time factor by realizing that silicon consists of a diamond crystal structure. In the case of the local condition hamper fast-response, crack stability is affected, leading to sharp instability events. The external surface slip traces examination on different crack orientations was very deductive. Firstly, in providing a physical meaning to the proposed model as related to dislocations emission from the crack-tip. For example, it was observed that the dominant slip system at the early stage of yielding was $\{112\}<111>$ and $\{011\}<111>$. This behavior became more complicated when multiple slip systems were activated that occurred at higher stress intensity levels. As shown in Fig. 4 by the Normaski contrast, more involved traces became visible at the 
crack-tip vicinity for the $\{001\}<110>$ orientation. The slip band traces were also supplemented by TEM observation. As related to dislocation structures, the crack-tip vicinity remained challenging. As shown in Fig. 5 the TEM image remained consistent, which the organized dislocation array emitted from the crack, tip. The TEM image was taken from the crack-tip vicinity of the $\{001\}<110>$ oriented sample. More information is elaborated in $[7,8]$.

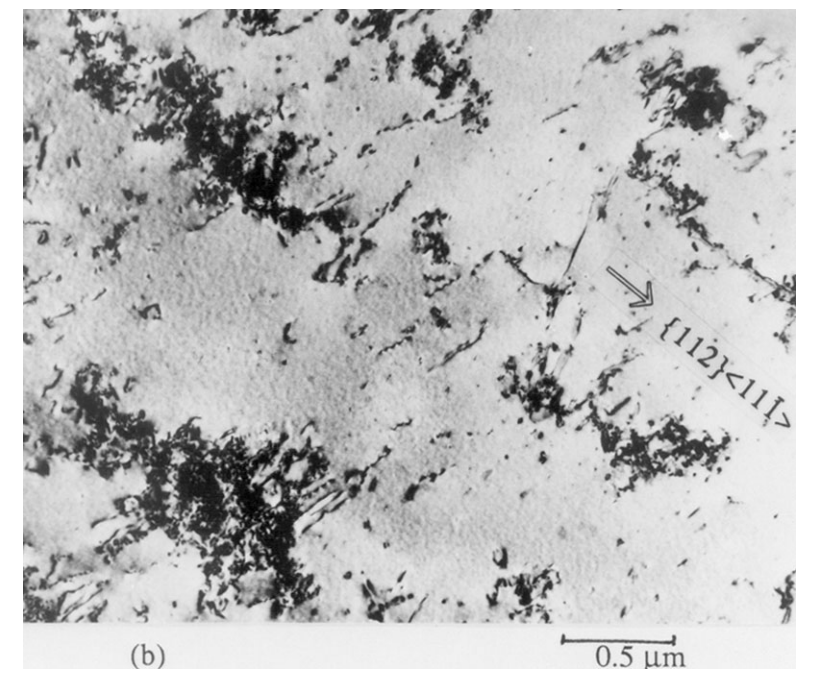

Figure 5: $\quad$ TEM indicated dislocation structure at the crack tip vicinity $\{001\}<110>$ oriented sample.

\section{Discussion}

The current model [4-9] followed the extended formulation by Atkinson and Clements [8], Lii et al [5] and Lii and Gerberich [9] superdislocation model. Basically, it has been founded on the crack tip dislocations interaction following a systematic theoretical/experimental program. On the other hand, the study confronted with the desire to establish the crack-tip mechanical environment, matched with consistent experimental confirmation. This viable experimental confirmation became more involved by considering complicated events, such as cleavage fracture, ductile/brittle transition and crack stability aspects with and without environment interaction. Accordingly, in the Fe-3\%wtSi single crystals the surface trace analysis also supported by TEM observations provided a sound physical base to the proposed model including crystal plasticity argumentations. In fact, crack-tip dislocations emission, their arrangement and distribution have been verified by TEM observations. However, in order to ease the numerical computation the present superdislocation model adopted a non-continuous distribution of the dislocation array. Moreover, the simulation assumed single dislocation emission from the crack-tip under local or for field plasticity. 
Following this procedure without excluding the possible role of external sources the crack-tip mechanical environment could be established. In addition, exploring the crack-tip shielding potential contributed to the significant increase of the local stress. The initial effects of localized plasticity beside the intensification of the crack-tip mechanical field might cause dramatic transition of the mechanical response. For example, circumstances for cleavage occurrence might become possible and even more so with environmental interactions. The cleavage phenomena in semi-brittle source models revealed additional major argument. The model as depicted in Fig.6 introduces the concept of the dislocation free zone. The crack-tip of relatively strong material, including the currently selected single crystals in mode I (open mode) indicated that the dislocation free zone is confined to $20 \mathrm{~nm}$. The important issue here is that fracture resistance includes an additional length scale emerging from the model. Some brief additional remarks are in order. The model introduced local $\mathrm{k}$ and global $\mathrm{K}$ stress intensity factor values. The local $\mathrm{k}$ value increases monotonically as the number of dislocations increases. For equilibrium requirement, which is necessary for the higher imposed global $\mathrm{K}$, this introduces criteria for cleavage in contrast to continuous dislocation emission. It also appears natural that the dislocation free zone decreases with $\mathrm{K}$, as mentioned, monotonic loading increases the global $\mathrm{K}_{\mathrm{I}}$ in an open mode case. The crack tips emit dislocations that shield the crack tip from cleavage. Locally, dislocation distribution modified the crack tip stress up to the value that might exceed the local Griffith value to promote cleavage. Nevertheless, the experimentally based findings remain consistent with crystal plasticity habits alluding to the critical role of the crack system orientation. The study of Spielmannova et al [10] has been centered on the crack orientation affecting the ductile-brittle behavior of $\mathrm{Fe}-3 \mathrm{wt} \% \mathrm{Si}$ single crystals. The study is atomistic or molecular dynamic simulation, based on the N-body interatomic potential of Finnis-Sinclair type by utilizing medium size samples containing more than $10^{6}$ atoms. In fact, the simulation indicated the crack-tip dislocation emission, brittle and ductile mechanical response and the loading rate domination. At least phenomenologically, the atomistic simulation appears consistent with the present investigation. Notice that the two models emerged from different approaches but provide the kind of mutual support and validation to the obtained findings. With respect to the ultra fine silicon particles, the present study also extended the unique interest in silicon to the issue of scale effects. As is known, silicon and silicon-based materials are dominant structural materials in microelectronics. As such, regardless of the scale, the life of silicon components subjected to static or cyclic load remains a major concern. Reliability assessment and a frequently higher degree of reliability are required in critical micro-scale devices. This becomes self-explained in sensor and transducer applications. The present novel experimental technique allowed a fine resolution regarding the irreversible displacement component. Moreover, under the current load state the model was based on dislocation dynamic effects. The role of dislocations has also been revealed by the occurrence of static and dynamic hardening that might contribute to the distinct arguments concerning the fatigue failure mechanism of silicon-based small volume materials. For the 
sake of briefness only some of the methodologies from the literature are finally described, realizing that the micro-components are subjected to mechanical and thermal cyclic loads. The main distinction in the proposed micromechanism has been related to the domination of dislocations in silicon at ambient temperature. The case study as addressed by Muhlstein et al [12] described more of an oxide layer domination affecting crack initiation and cyclic subcritical crack extension. The susceptibility to oxide growth and stress corrosion cracking is lacking generality. Damage accumulations are not only cyclic dependent, rather highly sensitive to the strain amplitude. Thus, from the design point of view, low cycle fatigue in silicon relies on the crack initiation stage. Cycles more than $10^{4}$ in silicon can be achieved only in low damage parameters. More progress in damage assessment might enable better prediction regarding the total life of small volume components and cyclic stress/strain conditions. Specifically, the current paper is constructed in describing some of the intensive activities in elastic-plastic crystalline single crystals. First, Fe-3\%wtSi single crystals were selected beside ultra fine silicon particles. An extended theoretical/experimental program has been followed that broadens the scope of interest beyond the strength issue. By utilizing pre-cracked specimens, fracture mechanics methodology could be applied, shedding light on mechanical related topics. These include fracture resistance, crack-tip dislocations interaction, crack stability, shielding potential and the ductile/brittle transition phenomena. Important insights associated to the aforementioned program have been addressed by Lii et al [5] and by Gerberich et al [4]. Secondly, regarding the small volume components section, the local approach was literally utilized due to geometrical constraint. In addition, silicon is normally defined to be relatively strong material that enabled the study of the role of the state of stress and the scale effects. Note that in the nano-section, mechanical tests have been conducted by contact. Again for the Fe-Si case the study also recognized simulations in 3D by molecular dynamic as addressed by Spielmannova et al [10] assisted by Finnis-Sinclair interatomic potentials. Regarding simulations, more is discussed concerning quasi-continuum models. Here, mainly the cracktip dislocation emission model was argued following Atkinson and Clements procedure [8] and also Lii and Gerberich [9] with a different dislocations arrangement.

\section{Summary and conclusions}

High performance materials progress relates to the major functional properties whether electrical, magnetic, optical or environmental. In all cases structural integrity is required and might be achieved only in a comprehensive way of understanding. Two examples have been elaborated, both in single crystal systems. The Fe-3wt\% $\mathrm{Si}$ description is centered on crack-tip dislocations source affecting the crack-tip mechanical environment. As such, in quasi-brittle materials ductile-brittle transitions might occur. Beside the crack length that has been introduced to be essential in the fracture resistance description, additional critical length scale emerged in terms of the dislocation-free zone concept. The 
ultra fine particles of silicon demonstrate the cyclic response beside the scale effects. The strength increase is size dependent and the role of dislocation activity also includes shielding potential that points to an important direction for developments. In small volume performances, wear and tribology explorations are striking. Thus the following was concluded.

(1) Phenomenologically, atomistic circulations in Fe-3wt\% $\mathrm{Si}$ single crystals and crack-tip dislocations source models remained consistent.

(2) Basically, the development of the crack-tip source model including the numerical computation intended to shed light on the cleavage events and crack stability aspects.

(3) In ultra fine silicon particles, contact mechanics methodology assisted exploration of the partition of reversible and irreversible displacements response, to be size dependent.

(4) Static and dynamic hardening behavior supported a line-defect analysis to be highly relevant even in the nano scale regime.

(5) In thin films of silicon at ambient temperatures the crack nucleation stage in fatigue actually establishes a design criterion in terms of crack nucleation life being a critical life prediction quantity.

\section{Acknowledgements}

Experimentally based findings were supported by the Chemical Engineering and Materials Science as well as the Mechanical Engineering Departments at the University of Minnesota that are acknowledged and highly appreciated.

\section{References}

[1] High strength material, ed. V. F. Zackay, Wiley New York, 1965.

[2] A. Kelly, Strong solids, Clarendon, Oxford, 1966.

[3] Rao N. P., Lee H. J, Kelkar M, Hansen D. J., Heberlein J. V. R., McMurray P.H., Girschick S.L. Nanosract. Mater. 9:129, 1997.

[4] Gerberich W. W., Mook, W. M., Perrey C. R., Carter C. B, Baskes M. I, Mukerjee R., Gidwani A., Heberlein J.V.R., McMurray P. H., Girshick S. L., J. Mech Phys Solids 51:979, 2003.

[5] Lii M. J. Chen X. F. Katz Y. and Gerberich W. W. Dislocation modeling and acoustic emission observation of alternating ductile/brittle events in Fe3\% Si crystals, Acta Metall mater. 38, 2435-53, 1990.

[6] Gerberich W. W., Oriani R. A., Lii M. J., Chen X. F. and Foceke T. The necessity of both plasticity and brittleness in fracture thresholds of iron, Phil. Mag. 63A, pp. 363-76, 1991.

[7] Chen X. F., PhD Thesis, University of Minnesota, Sep. 1989.

[8] Atkinson, C, and Clements D. L., The influence of anisotropy and crystalline slip on relaxation at a crack tip, Acta Metall 2, pp. 55-60, 1973.

[9] Lii M. J. and Gerberich W. W., The stress state close to a crack tip shielded by dislocation array, Ser. Metall, 22, pp. 1779-82, 1988. 
12 High Performance Structures and Materials IV

[10] Spielmannov. A., Landa M., Machova, A., Hansild P. and Lejcek P. Influence of crack orientation on the ductile-brittle behavior in $\mathrm{Fe}-3 \% \mathrm{wtSi}$ single crystals, Material Characterization, 2007.

[11] Zielinski W., Lii M. J., and Gerberich W.W. Crack-tip dislocation emission arrangement for localization in situ TEM observations. Acta Metall, Mater 40. pp. 2883-94, 1992.

[12] Muhlstein. C. L, Brown C. B. and Ritchie R. O., Sensors and actuators, A94, p. 178, 2001. 